\title{
Combination Lateral Orbitotomy and Orbit Decompression as a New Approach for the Treatment of Adhesion Cavernous Haemangioma in the Orbapex
}

\section{Chengyan Fang}

Second People's Hospital of Yunnan Province

Hai Liu

Second People's Hospital of Yunnan Province

Wenjia Zhang

Second People's Hospital of Yunnan Province

\section{Zhongkun Yang}

Second People's Hospital of Yunnan Province

\section{Yabing Yang}

Second People's Hospital of Yunnan Province

\section{Tao Tao}

Second People's Hospital of Yunnan Province

\section{Zhulin Hu}

Second People's Hospital of Yunnan Province

Kangwei Jiao ( $\square$ Kangwei.jiao@whu.edu.cn )

Second people's hospital of Yunnan province

\section{Research article}

Keywords: an orbital tip, adhesion cavernous haemangioma, lateral resuscitation, decompression, combined therapy

Posted Date: June 19th, 2020

DOI: https://doi.org/10.21203/rs.3.rs-36138/v1

License: (c) (i) This work is licensed under a Creative Commons Attribution 4.0 International License. Read Full License 


\section{Abstract}

Orbital cavernous haemangioma $(\mathrm{OCH})$ is a benign, slow-growing lesion of intracranial space. Especially, lesions located at the tip of the angle to form adhesions to the optic nerve that remains extremely challenging. This study investigated the clinical features and combined treatment of patients with $\mathrm{OCH}$ who underwent orbital incision or decompression and incision. This was a retrospective observational study. Between September 2015 and August 2018, Cases with pathologically confirmed orbital cavernous haemangioma were treated with either of two eyelid incision approaches. The clinical features and treatment outcomes were compared between groups. Forty-three patients with unilateral haemangioma involving adhesion of the temporal and optic nerves were included in this study. Of the 43 patients, $60 \%$ were women; the mean age was 40.2 years (range, 25-71 years). The main complaints were painless progressive exophthalmos and visual impairment. Complete removal of the tumor was achieved in all patients who underwent a standard lateral opening. Visual acuity (VA) was stable in 21 patients (91\%), whereas it worsened in 2 patients (8.6\%). Complete tumor removal was achieved in all patients who underwent lateral decompression. Visual acuity was initially stable in 20 patients (100\%); at one week postoperatively, visual acuity worsened in 1 patient (5\%). Multivariable analysis showed that tumor location $(p<0.001)$, the level of adhesions to the optic nerve $(p<0.001)$, and surgery options (correlation coefficient $r=-0.5, p<0.001$ ) were significantly associated with postoperative VA. Cavernous haemangioma with orbital tip and optic nerve adhesion was accurately diagnosed and localized by imaging examinations before surgery. Lateral opening and decompression combined with decompression effectively enlarged the orbital volume and reduced the internal pressure of the eyelid. Patients were followed up for at least six months with no recurrence after either surgical procedure

\section{Introduction}

Orbital cavernous haemangioma is the most common benign tumor originating in the septum in adults, constituting $14.82 \%$ of orbital tumors $[1,2]$. It is a vascular lesion that constitutes a well-defined hamartoma composed of irregular thick-walled and thin-walled sinusoidal vascular channels[2]. Although cavernous haemangioma is histologically benign, it is disabling and considered to be anatomically or positionally malignant due to compression of inner or adjacent structures[3]. In particular, haemangiomas located at the tip of the angle increase due to repeated bleeding and form adhesions to the optic nerve, thereby raising intraocular pressure through the increased volume; thus, surgical removal lesions at this location is exceptionally challenging.

The application of modern imaging techniques such as computed tomography (CT) and magnetic resonance imaging (MRI) has significantly improved the preoperative positioning and qualitative accuracy $[4,5]$, but there remain many deficiencies, such as the degree of adhesion of the tumor and assessment of the integrity of the capsule [6]. Therefore, the choice of surgical approach for orbital tumors requires accurate imaging and the reduction of surgical complications. 
At present, there are various surgical methods for the treatment of apical haemangioma; however, to date, research on $\mathrm{OCH}$ has focused on the classic lateral incision, transcranial approach, transconjunctival approach, supraorbital approach, and the transnasal endoscopic, etc. [7-10]. The traditional standard lateral open surgery method involves cutting the iliac crest and the superior or inferior iliac crest, which can expose the surgical field of vision and reduce the occurrence of comorbidities [11]. It is often applied to the side of the eyelid or small lesions at the base of the iliac crest. The supraorbital approach is used to remove lesions located on the back of the eyelid; the transcranial approach can remove large lesions located inside the optic nerve[12]. The transnasal endoscopic approach can remove smaller

lesions[13]. However, each of these methods has distinct advantages and disadvantages; these may include invasiveness and associated neurological complications, or incomplete removal. Lateral sacral decompression combined with decompression is advantageous because it allows complete removal of lesions located in the muscle cone, which exhibit adhesion to the optic nerve and are 15-30 mm in diameter; however, intraoperative and postoperative angle pressure is increased, thereby conferring risk of visual impairment.

Therefore, surgery and postoperative recovery remain challenging. However, combination lateral orbitotomy and orbit decompression for the treatment of cavernous haemangioma in the orbital apex may provide a new option for the treatment of cavernous hemangioma with massive adhesions around the optic nerve.

Orbital cavernous haemangioma is the most common benign tumor originating in the septum in adults, constituting $14.82 \%$ of orbital tumors $[1,2]$. It is a vascular lesion that constitutes a well-defined hamartoma composed of irregular thick-walled and thin-walled sinusoidal vascular channels[2]. Although cavernous haemangioma is histologically benign, it is disabling and considered to be anatomically or positionally malignant due to compression of inner or adjacent structures[3]. In particular, haemangiomas located at the tip of the angle increase due to repeated bleeding and form adhesions to the optic nerve, thereby raising intraocular pressure through the increased volume; thus, surgical removal lesions at this location is exceptionally challenging.

The application of modern imaging techniques such as computed tomography (CT) and magnetic resonance imaging (MRI) has significantly improved the preoperative positioning and qualitative accuracy $[4,5]$, but there remain many deficiencies, such as the degree of adhesion of the tumor and assessment of the integrity of the capsule [6]. Therefore, the choice of surgical approach for orbital tumors requires accurate imaging and the reduction of surgical complications.

At present, there are various surgical methods for the treatment of apical haemangioma; however, to date, research on $\mathrm{OCH}$ has focused on the classic lateral incision, transcranial approach, transconjunctival approach, supraorbital approach, and the transnasal endoscopic, etc. [7-10]. The traditional standard lateral open surgery method involves cutting the iliac crest and the superior or inferior iliac crest, which can expose the surgical field of vision and reduce the occurrence of 
comorbidities [11]. It is often applied to the side of the eyelid or small lesions at the base of the iliac crest. The supraorbital approach is used to remove lesions located on the back of the eyelid; the transcranial approach can remove large lesions located inside the optic nerve[12]. The transnasal endoscopic approach can remove smaller

lesions[13]. However, each of these methods has distinct advantages and disadvantages; these may include invasiveness and associated neurological complications, or incomplete removal. Lateral sacral decompression combined with decompression is advantageous because it allows complete removal of lesions located in the muscle cone, which exhibit adhesion to the optic nerve and are $15-30 \mathrm{~mm}$ in diameter; however, intraoperative and postoperative angle pressure is increased, thereby conferring risk of visual impairment.

Therefore, surgery and postoperative recovery remain challenging. However, combination lateral orbitotomy and orbit decompression for the treatment of cavernous haemangioma in the orbital apex may provide a new option for the treatment of cavernous hemangioma with massive adhesions around the optic nerve.

\section{Materials And Methods}

\section{Participants}

This study was approved by the Medical Ethics Committee of Second People's Hospital of Yunnan Province. Informed consent for the surgical procedures, obtained from all patients, was written. This study included all patients referred from September 2015 to August 2018, all 43 patients with a diagnosis of $\mathrm{OCH}$ were treated in our hospital after surgery by histopathology. The standard lateral opening was performed in 23 patients (54\%), while lateral decompression combined with decompression was performed in 20 patients (46\%).

\section{Preoperative Evaluations}

Preoperative eye examinations included assessment of visual acuity (VA; Snellen chart distance of 6 meters), eye movement, stereo vision, and pupillary reflex. Besides, the following evaluations were performed: eyeball protrusion measurement (Hertel eyeball prominence), visual field test (Humphrey or Goldman Perimetry), non-contact tonometry (NT-510, NIDEK, Japan) for fundus examination of intraocular pressure and direct ophthalmoscopy (B-ultrasound (MD-2400S, China) for examination of post-ocular condition;

computed tomography (Aquilion ONE, Toshiba), Magnetic Resonance Imaging (GE Optima MR360 1.5T, US)

Computed tomography (CT) and magnetic resonance imaging (MRI) evaluations were performed as follows (Figure 1): clear borders, rounded muscles, and unclear borders between the coronal tumor and the optic nerve in all patients. Contrast enhancement showed a blood vessel in the posterior pole and 
revealed that the tumor was irregular or mulberry-like in shape. Contrast enhancement showed that the posterior pole was burr-like, suggesting that the optic nerve exhibited different degrees of adhesion. Orbital ultrasonography was performed in all patients to confirm the diagnosis of the tumor and the location of the lesion. A subset of patients underwent orbital ultrasonography using type $A$ and type $B$ ultrasound $(10 \mathrm{MHz})$ to confirm the diagnosis of the tumor and the location of the lesion.

\section{Surgical Indications and Approaches}

Surgical indications for patients with clinical signs of intraorbital lesions included the following: (1) visual impairment (Snellen chart visual acuity of less than $6 / 7.5)$; (2) progressive unilateral exophthalmos (1.5 mm larger than the contralateral eye); (3) double vision; (4) OCH - related orbital pain; (5) choroidal folds. Tumors were located in the tip of the iliac crest. Based on the results of computed tomography or magnetic resonance imaging, 43 patients underwent lateral resection of the tumor: 23 received standard lateral resuscitation, and 20 received lateral resuscitation combined with decompression surgery.

\section{Surgical Approaches}

The standard lateral orbitotomy approach was as follows: (1) the incision was extended to the affected side, $1 \mathrm{~cm}$ from the eyelashes; (2) the periosteum was incised along the outer edge of the iliac crest; (3) the exposed rim outer wall was separated, and the iliac crest was cut with a bone saw; (4) a cut was made parallel to the upper and lower edges of the file.

The lateral opening combined with decompression approach was as follows (Figure 2): (1) standard lateral opening was performed; (2) the outer wall of the angle was clamped; (3) a bite clamp was used in combination with grinding to remove the lateral side of the angle; (4) when the bone was deep in the wall, it was ground to the meninges of the temporal lobe, the lateral wall of the upper iliac cleft was opened, and part of the bone of the lacrimal gland was removed to enlarge the sacral cavity; (5) the operation was carefully monitored to prevent the leakage of cerebrospinal fluid.

Both surgical procedures were performed under general anaesthesia. Postoperative histopathological examination confirmed the diagnosis of cavernous haemangioma in all patients. The improvement of primary complaints was regarded as the postoperative outcome. All patients were followed up for at least six months.

\section{Statistical Analysis}

All statistical analyses were performed by SPSS (IBM Corp. Released 2014. IBM SPSS Statistics for Windows, Version 23.0. Armonk, NY: IBM Corp.). To present data, we used to mean, standard deviation (SD), median, and range. Adobe Illustrator CC 2019 software CS3 (Adobe Systems Incorporated, San Jose, CA) was used for primary image processing. A comparison of means and medians was made using Student's $t$-test or ANOVA, as required. Correlation techniques were used to analyse associations 
between variables (Pearson or Spearman, according to normality). All tests were two-sided, and $p$ values $<0.05$ were considered statistically significant.

\section{Results}

The 43 patients in this study included male $(n=17,40 \%)$ and female $(n=26,60 \%)$ (Mean age, 40.2 years; range, from 25 to 71 years). Right eyes ( $n=19,44 \%$ ) and left eyes ( $n=24,56 \%)$ were included. Clinically, the patients had acute or subacute episodes (e.g., eyeball protrusion or worsened vision) with an average duration of 5 years (range, from 10 days to 30 years). Symptoms and signs are presented in Tables 1 and 2.

After excision of the tumor, the postoperative outcomes and complications are shown in Tables 3 and 4 . At six months postoperatively, all cases $(n=43,100 \%)$ mean exophthalmometry was decreased $5.1 \pm 1.2$ $\mathrm{mm}(p<0.001)$ and all cases developed enophthalmos of $1.2 \pm 0.2$ (range 1-3) millimetre, BCVA in the cases with combination therapy $(n=5,26 \%)$ and with lateral orbitotomy $(n=5,26 \%)$ have been improved, with a mean (SD) of 2.1 and 3.0 letters in both of the groups, respectively, adjusted mean difference, $0.6(95 \% \mathrm{Cl},-2.3$ to 3.4$) ; p=0.31$. The rest of the other cases had no significant differences in visual acuity relative to the preoperative values. No optic nerve damage occurred (Figure 3).

Tumour location appeared to be significantly associated with BCVA after surgery $(r=-0.4, p=0.01)$; Preoperative BCVA showed a strong positive correlation with postoperative BCVA at six months ( $r=$ $+0.61, p<.001)$, However, there were no significant differences for postoperative BCVA between the two surgery techniques $(p=0.285)$ after six months of follow up. We also noted two cases of vision loss of the lateral orbitotomy due to surgical trauma (Table 4).

Of the 32 patients with exophthalmos ( $n=17,40 \%$ in Comb.; $n=15,35 \%$ in LO), all 32 patients $(100 \%)$ had complete resolution of exophthalmos. Of the 40 patients in BCVA loss ( $n=19,44 \%$ in Comb.; $n=19$, $44 \%$ in LO), 9 (43.0\%) had improvement in comb group, 5 (26\%) had improvement in LO group. While patients in both of the two groups (patient $12,57 \%$ in Comb. and patient $n=12,74 \%$ in LO) who had longstanding optic neuropathy with no improvement in BCVA. Of the 38 patients with visual field defect $(\mathrm{n}=$ $18,47 \%$ in Combination; $n=20,53 \%$ in LO), $5(28 \%)$ had complete resolution in Combination, only 7 (11\%) had complete resolution and $12(36 \%)$ had partial improvement in LO, while 11 (55\%) remained unchanged due to long-standing compressive optic neuropathy. $100 \%$ had improvement in headache. Of the 12 patients in diplopia, $n=3(44 \%)$ had complete resolution of diplopia in Comb, while $n=3(62 \%)$ had complete and partial resolution of the diplopia, respectively.

$\mathrm{CRAO}=$ central retinal artery occlusion; $\mathrm{ONI}=$ optic nerve injury Combination $=$ lateral orbitotomy + decompression orbitotomy

\section{Discussion}


This study introduces a new technique for dissection of $\mathrm{OCH}$ with a meager complication rate in our center in a long period. In agreement with previous studies, there was no difference in the frequency of both side involvements. The ratio of gender in the current series was about 1 / 1.5 (M / F); none of these cases in our study had any systemic involvement.

Compared with previous studies which axial proptosis was the main complaint. The chief of the complaint was visual impairment in $93 \%$ of our cases. It is interesting that visual

impairment was not directly related to proptosis and indicates that vision damage may be related to the level of adhesion between optic nerve and lesions, and the location of $\mathrm{OCH}$. It is not necessarily manifested as exophthalmos.

Different approaches to the $\mathrm{OCH}[9,11-13]$, which reside in the very compact space of the orbit, have been reported decades. However, in the case of severe $\mathrm{OCH}$, particularly with apical $\mathrm{OCH}$ causing adhesion of optic neuropathy is still facing many problems that need to be solved. Because of often tenacious attachments to fixed apical structures, even careful surgical removal can imperil vision.

Although the lateral orbitotomy is concerning the most effective and safest approach for $\mathrm{OCH}$, we encountered 2 cases with vision worsening ( 1 is CRAO and ONI is another) after lateral orbitotomy in 6 months later. We believe this cause for vision worsening in our series that is the way with LO only the anterior surface of the tumor is manipulated, the posterior parts, especially for non-visualized area may have dense attachments and adhesions to surrounding optic never may become damaged during removal, even leading to incomplete excision due to the narrow visual field of operation. In such cases of tight adhesion between the tumor and the optic nerve where surgical risks may be considered too high, the alternative option of combination of lateral orbitotomy and decompression orbitotomy dissection can be regarded as for its beneficial effects of remove of tumor completely, improvement of visual acuity, exposed field of vision clearly and resolution of visual field defect without recurrence of tumor or other serious complications after closely monitored 6-month follow-up.

However, a subset of patients who had undergone combination therapy continued to exhibit visual field defects at six months of follow-up; this complication was more frequent in patients treated with the simple lateral opening. We presume that, although tumor lesions were removed entirely, the long-term compression by tumors before surgery caused irreversible damage to nerve fibers. Double vision was reported during follow-up at six

months after surgery in both groups; this was more frequent in patients treated with the simple lateral opening. Ptosis was also more prevalent in patients treated with the simple lateral opening. Besides, pupil dilation occurred in a substantial proportion of the patients; the frequency was similar between groups. In all patients with pupil dilation, the tumor was more massive, mostly located near the temporal and ciliary ganglia of the optic nerve. 
Since it is a retrospective study and the limitation of our study are long-term (for years) observation and visual function tests results, including VEP, color vision tests that could better assess the presence of compressive optic neuropathy and evaluate its changes after surgery.

In summary, the selection of the appropriate surgical approach is essential for the successful treatment of cavernous orbital hemangioma. For patients with haemangioma who have obvious optic nerve adhesion and high sacral pressure, simple lateral sacral surgery should be performed with caution. Furthermore, it has shown to be safe, with no complications associated with combination surgical resection -related nor any tumor removing-related visual morbidity.

Thus, we suggest employing the technique of lateral orbitotomy combined with decompression may constitute a more appropriate choice of such lesions.

\section{Declaration}

\section{Availability of data and materials}

The datasets generated and/or analysed during the current study are not publicly available due to protecting individual patient privacy but are available from the corresponding author on reasonable request.

\section{Competing interests}

The authors declare that they have no competing interests.

\section{Authors' contributions}

Author Zhulin Hu designed the study and Kangwei Jiao wrote the initial draft of the manuscript. Author Chengyan Fang contributed to the analysis and interpretation of data, and assisted in the preparation of the manuscript. All other authors contributed to data collection and interpretation, and critically reviewed the manuscript. All authors approved the final version of the manuscript, and agree to be accountable for all aspects of the work in ensuring that questions related to the accuracy or integrity of any part of the work are appropriately investigated and resolved.

\section{Consent for publication}

All authors have read and approved the contents of the manuscript, and the submission is not under review at any other publication.

\section{Ethics declarations}

\section{Ethics approval and consent to participate}


This study was approved by the Medical Ethics Committee of Second People's Hospital of Yunnan Province. Informed consent for the surgical procedures, obtained from all patients, was written.

Consent for publication

Written informed consent for publication of the participant clinical details was obtained from each patient. The patients and guardians of the participants that were minors at the time of the study $(<18)$ gave written consent for their respective children's personal or clinical details along with any identifying images to be published in this study.

Acknowledgements

None

Funding

None

\section{References}

1. Rootman DB, Heran MK, Rootman J, White VA, Luemsamran P, Yucel YH: Cavernous venous malformations of the orbit (so-called cavernous haemangioma): a comprehensive evaluation of their clinical, imaging and histologic nature. Br J Ophthalmol 2014, 98(7):880-888.

2. McNab AA, Wright JE: Cavernous haemangiomas of the orbit. Aust NZ J Ophthalmol 1989, 17(4):337-345.

3. Yan J, Cai Y, Wu Z, Han J, Pang Y: Cavernous hemangioma of the bony orbit. Yan ke xue bao = Eye science / "Yan ke xue bao" bian ji bu 2005, 21(3):147-151.

4. Jayaram A, Cohen LM, Lissner GS, Karagianis AG: A retrospective review of cases preoperatively diagnosed by radiologic imaging as cavernous venous malformations. Orbit 2017, 36(3):128-134.

5. Ohtsuka $\mathrm{K}$, Hashimoto $\mathrm{M}$, Akiba $\mathrm{H}$ : Serial dynamic magnetic resonance imaging of orbital cavernous hemangioma. American journal of ophthalmology 1997, 123(3):396-398.

6. Tian YM, Xiao LH, Gao XW: Adhesion of cavernous hemangioma in the orbit revealed by CT and MRI: analysis of 97 cases. Int J Ophthalmol 2011, 4(2):195- 198.

7. Harris GJ: Cavernous hemangioma of the orbital apex: pathogenetic considerations in surgical management. American journal of ophthalmology 2010, 150(6):764-773.

8. Calandriello L, Grimaldi G, Petrone G, Rigante M, Petroni S, Riso M, Savino G: Cavernous venous malformation (cavernous hemangioma) of the orbit: Current concepts and a review of the literature. Surv Ophthalmol 2017, 62(4):393-403.

9. Scheuerle AF, Steiner HH, Kolling G, Kunze S, Aschoff A: Treatment and long-term outcome of patients with orbital cavernomas. American journal of ophthalmology 2004, 138(2):237-244. 
10. Papalkar D, Francis IC, Stoodley M, Kaines A, Sharma S, Kalapesi FB, Wilcsek GA: Cavernous haemangioma in the orbital apex: stereotactic-guided transcranial cryoextraction. Clin Exp Ophthalmol 2005, 33(4):421-423.

11. Moufarreh M, Benbouzid MA, Ghailan MR, Chefchaouni M, Essakalli L, Kzadri M: [Removal of lateral orbital wall for management of orbital cavernous hemangioma]. J Fr Ophtalmol 2014, 37(1):18-22.

12. Brusati R, Goisis M, Biglioli F, Guareschi M, Nucci P, Gianni AB, Miglior S: Surgical approaches to cavernous haemangiomas of the orbit. Br J Oral Maxillofac Surg 2007, 45(6):457-462.

13. Gazioglu N, Abuzayed B, Tanriover N: Neuronavigation-guided endoscopic endonasal excision of an intraorbital intraconal cavernous hemangioma. J Craniofac Surg 2011, 22(5):1802-1805.

\section{Tables}

Table 1: Presenting symptoms in 43 patients with cavernous orbital haemangioma

\begin{tabular}{|ll|}
\hline Symptoms & No. of patients (\%) \\
\hline Visual impairment & $40(93 \%)$ \\
\hline Visual field defect & $38(88 \%)$ \\
\hline Exophthalmos & $32(74 \%)$ \\
\hline Orbital tension & $29(67 \%)$ \\
\hline Fundus disorders & $23(53 \%)$ \\
\hline Ocular motility disorders & $20(47 \%)$ \\
\hline Diplopia & $12(27 \%)$ \\
\hline Headache & $9(21 \%)$ \\
\hline
\end{tabular}

Table 2: Presenting signs in 43 patients with cavernous orbital haemangioma

\begin{tabular}{|lll|}
\hline Signs & \multicolumn{2}{l|}{ No. of patients (\%) } \\
\hline Visual impairment & 40 & $(93)$ BCVA: NLP - 4.9 \\
\hline Visual field defects & 38 & $(88 \%)$ \\
\hline Exophthalmos & 32 & $(74 \%) 3.6 \mathrm{~mm} \pm 0.8 \mathrm{~mm}$ \\
\hline Ocular motility disorders & 20 & $(47 \%)$ \\
\hline Optic atrophy & $16 \quad(37 \%)$ \\
\hline RAPD $(+)$ & $8(19 \%)$ \\
\hline Optic disc oedema & $7(16 \%)$ \\
\hline
\end{tabular}


BCVA = best-corrected visual acuity; NLP = no light perception; RAPD = relative afferent pupillary defect

Table 3: Results after removal of cavernous orbital haemangioma $(n=53)$

\begin{tabular}{|c|c|c|c|c|c|c|}
\hline \multirow[t]{4}{*}{ Signs } & \multirow{2}{*}{\multicolumn{2}{|c|}{$\begin{array}{l}\text { Patients affected } \\
\text { preoperatively }\end{array}$}} & \multirow{2}{*}{\multicolumn{2}{|c|}{$\begin{array}{l}\text { Improvement at one } \\
\text { week postoperatively }\end{array}$}} & \multirow{2}{*}{\multicolumn{2}{|c|}{$\begin{array}{l}\text { Improvement at six } \\
\text { months postoperatively }\end{array}$}} \\
\hline & & & & & & \\
\hline & Combination & LO & Combination & LO & Combination & LO \\
\hline & 17 & 15 & 17 & 15 & 17 & 15 \\
\hline \multicolumn{7}{|c|}{ Exophthalmos } \\
\hline & $(40 \%)$ & (35\%) & $(100 \%)$ & $(100 \%)$ & $(100 \%)$ & $(100 \%)$ \\
\hline & 21 & 19 & 5 & 3 & 9 & 5 \\
\hline \multicolumn{7}{|l|}{ BCVA } \\
\hline & $(49 \%)$ & $(44 \%)$ & $(24 \%)$ & $(16 \%)$ & $(43 \%)$ & $(26 \%)$ \\
\hline Visual field & 18 & 20 & 12 & 13 & 5 & 2 \\
\hline defect & $(47 \%)$ & $(53 \%)$ & $(67 \%)$ & $(65 \%)$ & $(28 \%)$ & $(10 \%)$ \\
\hline Diplopia & 7 & 5 & 1 & 1 & 3 & 3 \\
\hline$(16 \%)$ & $(12 \%)$ & $(14 \%)$ & $(20 \%)$ & $(43 \%)$ & $(60 \%)$ & \\
\hline \multicolumn{7}{|l|}{ Ocular } \\
\hline 13 & 7 & 2 & 2 & 6 & 5 & \\
\hline \multicolumn{7}{|l|}{ motility } \\
\hline$(30 \%)$ & $(16 \%)$ & $(15 \%)$ & $(29 \%)$ & $(46 \%)$ & $(71 \%)$ & \\
\hline \multicolumn{7}{|l|}{ disorders } \\
\hline 6 & 3 & 1 & 1 & 5 & 3 & \\
\hline \multicolumn{7}{|l|}{ Headache } \\
\hline$(14 \%)$ & $(7 \%)$ & $(17 \%)$ & (33\%) & $(100 \%)$ & $(100 \%)$ & \\
\hline
\end{tabular}

Combination = lateral orbitotomy + decompression orbitotomy; LO = lateral orbitotomy;

Table 4: Complications after removal of orbital cavernous hemangioma $(n=43)$ 


\begin{tabular}{|c|c|c|c|c|c|}
\hline \multirow[t]{2}{*}{ Complication } & \multicolumn{2}{|c|}{1 week postoperatively } & \multicolumn{3}{|c|}{6 months postoperatively } \\
\hline & Combination & LO & Con & tion & LO \\
\hline & 1 & 2 & 0 & & 2 \\
\hline \multicolumn{6}{|c|}{ Vision worsening } \\
\hline & $(5 \%)$ & $(8.6 \%)$ & $(0 \%$ & & $(8.6 \%)$ \\
\hline & 19 & 19 & 20 & 21 & \\
\hline \multicolumn{6}{|c|}{ Vision stabilising } \\
\hline & $(95 \%)$ & $(83 \%)$ & $(100 \%)$ & $(91 \%)$ & \\
\hline Vision Loss & 0 & 3 & 0 & 2 & \\
\hline \multirow[t]{2}{*}{ (CRAO; ONI) } & $(0 \%)$ & $(13 \%)$ & $(0 \%)$ & $(8.6 \%)$ & \\
\hline & 7 & 8 & 2 & 6 & \\
\hline \multicolumn{6}{|l|}{ Pupil dilation } \\
\hline & $(35 \%)$ & $(36 \%)$ & $(10 \%)$ & $(27 \%)$ & \\
\hline Visual field & 6 & 8 & 2 & 3 & \\
\hline defect & $(31 \%)$ & $(36 \%)$ & $(8.6 \%)$ & $(19 \%)$ & \\
\hline Ocular motility & 5 & 5 & 2 & 4 & \\
\hline \multirow[t]{2}{*}{ disorders } & $(25 \%)$ & $(30 \%)$ & $(10 \%)$ & $(17 \%)$ & \\
\hline & 15 & 16 & 1 & 3 & \\
\hline \multicolumn{6}{|l|}{ Diplopia } \\
\hline & $(75 \%)$ & $(70 \%)$ & $(5.0 \%)$ & $(13 \%)$ & \\
\hline & 13 & 18 & 2 & 4 & \\
\hline \multicolumn{6}{|l|}{ Blepharoptosis } \\
\hline & $(65 \%)$ & $(78 \%)$ & $(10 \%)$ & $(17 \%)$ & \\
\hline & 19 & 20 & 0 & 1 & \\
\hline \multicolumn{6}{|l|}{ Headache } \\
\hline & (95\%) & $(87 \%)$ & (0\%) & $(4.3 \%)$ & \\
\hline
\end{tabular}

$\mathrm{CRAO}=$ central retinal artery occlusion; $\mathrm{ONI}=$ optic nerve injury $;$ Combination = lateral 
orbitotomy + decompression orbitotomy

Figures
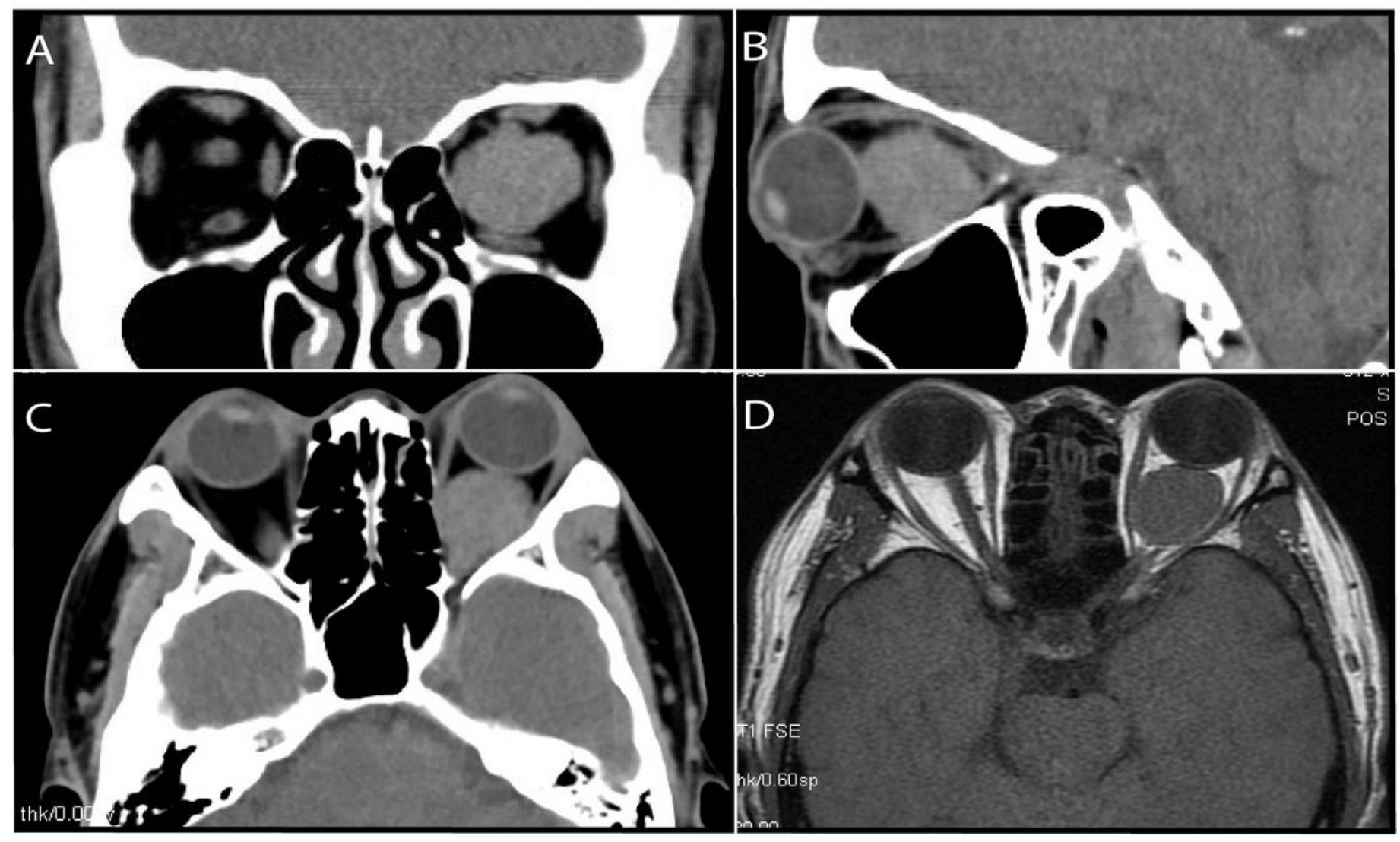

Figure 1

Figure 1 


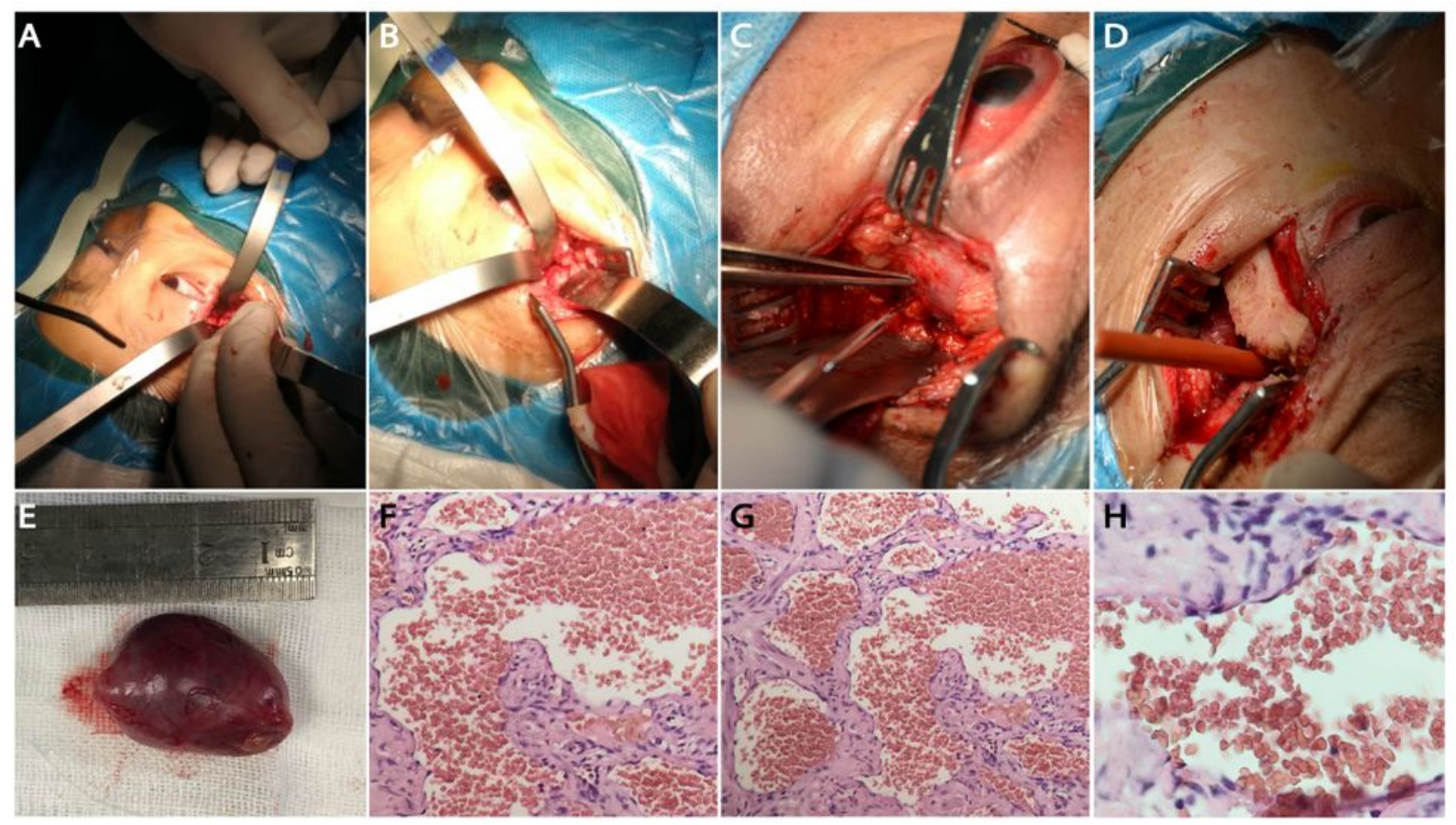

Figure 2

Figure 2
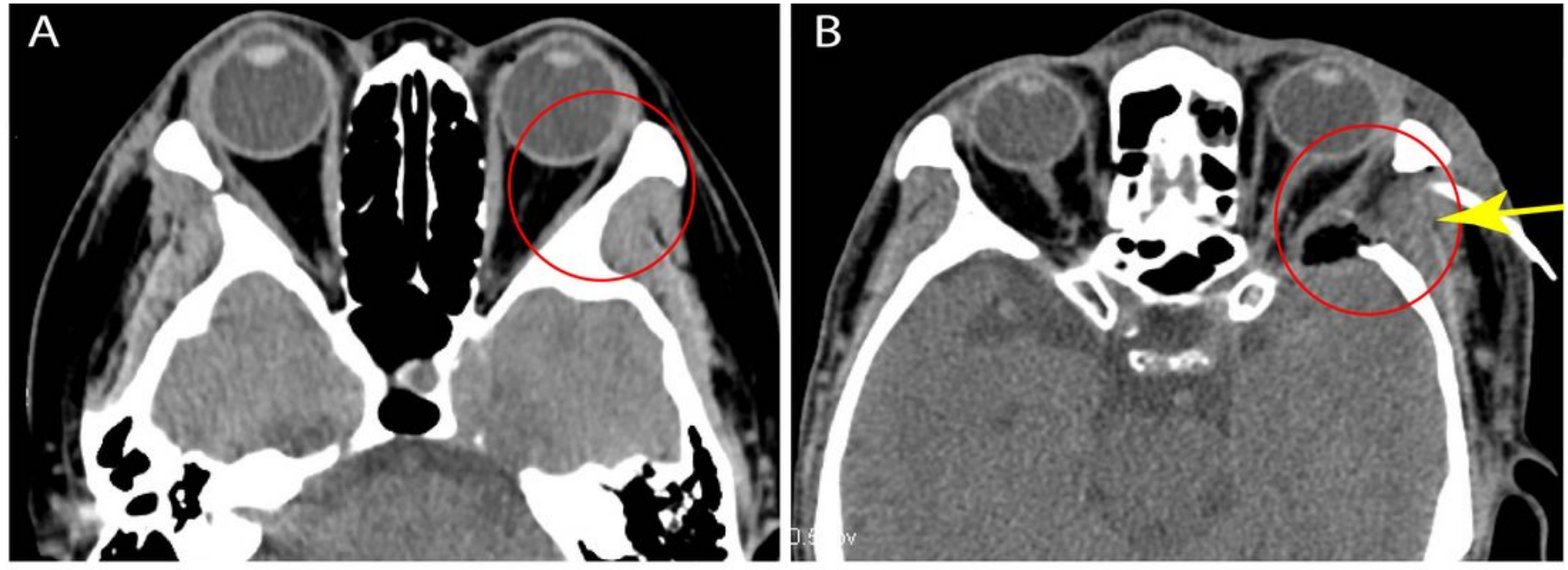

Figure 3

Figure 3 